\title{
Paper Technology und Wissensgeschichte
}

\author{
Volker Hess und J. Andrew Mendelsohn
}

1. A Cash-booke 2. A Write or Acquittance Booke 3. a booke for charges Merchandize 4. a Coppie booke of Letters 5. a Remembrance or Note Booke 6. a ffreight booke 7. a booke of Inv[en] toires sent 8. a booke of Inv[en] toires received [...] 14. a Wast [e] or day booke 15. a Journall 16. a Lidger [i.e. Ledger] 17. a Quadaranecra [i.e. quadernàccio, a rough work book] of Goods rec[eive]d \& cons[igne]d 18. a Custome-house booke 19. a booke of Cargoes of ships arrived \& dep[ar]ted 20. a Month booke 21. a booke et Coppie in partitos.

Robert Williams: Notes Concerning Trade, 1632-1654

(zit. nach Soll 2010: 368).

Es ist kaum vorzustellen, wie schwer bepackt man sich in der frühen Neuzeit auf die Reise begab. Nicht weniger als 21 verschiedene Formen von Notizbüchern sah dieser englische Kaufmann als notwendig an, um seine Angelegenheiten unterwegs in Ordnung zu halten. Solche Empfehlungen waren nicht ungewöhnlich. Fast immer wurden Notizbücher und Kladden herangezogen, wenn es galt, das aufzuschreiben, was man sah, las, kaufte, berechnete, überlegte oder ersann. So hielt ein deutscher Naturforscher seine Forschungsreise durch Sibirien (1720-1727) in vier verschiedenen Aufschreibeformaten fest, die alle auf die doppelte Buchführung rekurrierten (te Heesen 2005a). Auch Ulisse Aldrovandi griff, um seine Aufschriebe zu ordnen, auf die Technik der Alphabetisierung zurück, die er als Lehrling bei Kaufleuten gelernt hatte (Krämer 2013). Anders wäre es dem italienischen Lehrer der Naturgeschichte kaum möglich gewesen, die Abertausenden von Schnipseln, mit denen er die bekannte Natur in 83 dicken Folianten verzettelte, unter Loci communes, die Überschriften oder Schlagworte einer topischen Ordnung, zu bringen. ${ }^{1} \mathrm{Ab}$ einer gewissen Größenordnung ist die alphabetische Anordnung einfacher.

Die Frühe Neuzeit kannte außerordentlich viele Formen der Verschriftlichung. ${ }^{2}$ Was immer man mit Stift und Papier anstellen konnte, wurde ausprobiert. Und was sich als brauchbar für den einen Zweck erwies (zum Beispiel zur Aufstellung von Soll und Haben), wurde bald auch für andere 
verwendet (zum Beispiel Bills of Mortality siehe Rusnock 2002: 18). Im Mittelpunkt dieses Themenheftes der NTM steht die Frage: Wie gaben solche Schreib- und Papiertechniken dem Wissen von der Natur seine Form? Eine erschöpfende Antwort werden die vier Fallstudien zwar kaum geben können, doch möchten wir versuchen, auf drei historiographische Perspektiven aufmerksam zu machen, die sich für die weitere Erforschung von Papiertechniken anbieten.

\section{Epistemische Effekte}

Die Form des Aufschreibens zeitigt Wissen. Damit ist nicht gemeint, dass Wissen nur als Geschriebenes festgehalten und vermittelt werden kann. Vielmehr produzieren Aufschreibeformate in einem anderen sozialen oder funktionalen Zusammenhang als ihrem ursprünglichen epistemische Effekte, weil sie das Aufgeschriebene auf eine neue und meist unvorhergesehene Weise präsentieren. Das mögen jene Spezies des leipyrischen Fiebers sein, die François Boissier de Sauvages bei der Auflistung der Krankheitsbenennungen nach botanischer Weise entdeckte (Hess/Mendelsohn 2013), oder die Arbeit an den Kategorien seiner Naturlehre, zu der sich Aldrovandi bei der Alphabetisierung seiner Exzerpte gezwungen sah. Seine Zentauren wiederum sind ein wunderbares Beispiel dafür, wie das alphabetische Arrangement der zusammengetragenen Beobachtungen zwei- und vierbeiniger Pferdemenschen zuerst eine kritische Revision und schließlich eine Neudefinition dieses „Naturobjekts“ nach sich zog, mit der alle gefundenen Textstellen erfasst werden sollten (Krämer 2013). Heute mag man eine Naturgeschichte solcher Fabelwesen für den Ausweis wissenschaftlicher Naivität halten. Dies wird der Zusammenstellung aber nicht gerecht, in der die gelehrte Leserschaft säuberlich aufgelistet alle autoritativen Stellungnahmen für und wider die Existenz dieser Wunderwesen präsentiert bekam. Sie ist einerseits mehr als eine beliebige Sammlung von Beobachtungen, erhebt andererseits aber nicht den Anspruch auf eine theoretische Erörterung. Die auflistende Gegenüberstellung der positiven und negativen Stellungnahmen gleicht der kaufmännischen Buchführung in Soll und Haben. Diese Form des Listens bringt das Aufgeschriebene in einen neuen Zusammenhang. Die Naturgeschichte des Zentauren als Buchhaltung der befürwortenden und ablehnenden Beobachtungen zu präsentieren, erzeugt als solche eine andere Lesart und subsumierende Interpretation der geläufigen Beobachtungen. Diese Erzeugung, Vermittlung und Verbreitung neuen Wissens meinen wir, wenn wir von epistemischen Effekten des Aufschreibens und Ordnens reden.

Papier und Wissen, hard und soft: Form und Technik solcher Aufschriebe sind gleichermaßen materiell und ideell, was die Geschichtsschreibung seit 
einigen Jahren mit dem Begriff der paper technology zu beschreiben versucht hat (te Heesen 2005b). Wir verstehen hierunter die Summe aller Schreibverfahren (wie Listen, Formulare), Text- (wie Exzerpt, Index) und Papiertechniken (wie Karteikarten, Bandakten) und der damit verbundenen Werkzeuge (wie Stifte, Klebstoff, Schere), die (beabsichtigt wie unbeabsichtigt) beim Festhalten, Sammeln, Akkumulieren von (direkt oder vermittelt) Gesehenem und Bedachtem eingesetzt werden. ${ }^{3}$ Möglicherweise ist dieser Versuch einer Definition zu umfassend und zu unspezifisch, da er alle Formen des Aufschreibens einschließt. Eine spezifischere Begriffsbestimmung hat die Geschichtsschreibung der Wissenschaften, Technik und Medizin für ihren Bereich also erst noch zu leisten.

$\mathrm{Zu}$ dieser Begriffsschärfung soll das Themenheft beitragen. Diese Aufgabe verlangt auch die Eingrenzung dessen, was mit paper technology ${ }^{4}$ nicht gemeint ist, worüber sich angesichts der Forschungen des letzten Jahrzehnts streiten ließe. Um den Begriff der paper technology schärfer zu fassen, möchten wir vorschlagen, hiervon zu unterscheiden:

1. Paper tools, das heißt gezielt entwickelte und zweckgerichtet eingesetzte Werkzeuge auf Papier oder anderen Schriftträgern (Klein 2001). Sie stellen eine Art von technology of theory (siehe Warwick 2003) dar, wie am Beispiel chemischer Formeln oder logischer Symbole (Krämer 1988) gezeigt wurde. Hierzu lassen sich auch Diagramme (Edgerton 1980, Mahoney 1985) und andere Repräsentationstechniken (Lynch/Woolgar 1990, Kusukawa 2012) zählen.

2. Literary technology, das heißt all jene literarischen und rhetorischen Techniken, die gezielt zur Herstellung von Evidenz, Objektivität und Autorität eingesetzt werden (Shapin 1984, Dear 1985). Auf diesen intentionalen Einsatz hebt die Forschung zur literary technology ab (Dear 1991). Das gilt auch für die Interpretation klassischer Nomenklaturen wie Wübben 2009 und andere argumentieren.

\section{Wissensgeschichte und Allgemeingeschichte}

Eine begriffliche Präzisierung kann helfen, das historiographische Potential einer Wissensgeschichte der paper technology herauszuarbeiten. Denn eine Fokussierung auf Papiertechniken bettet über den Weg der Analyse von Aufschreibeformaten, Aufzeichnungspraktiken und der dabei eingesetzten Geräte und Materialien die in der Fachgeschichtsschreibung im Mittelpunkt stehenden epistemischen Praktiken in eine allgemeine Geschichtsschreibung ein. Auch wenn dies der Begriff der Papiertechniken vielleicht nahelegt, ist hierbei weniger an die Geschichte des Buchs und Buchdrucks und die ökonomischen, sozialen 
und kulturellen Folgen ihrer Einführung zu denken. ${ }^{5}$ Vielmehr geht es um das Zirkulieren der Aufschreibe- und Papiertechniken, die sich die humanistische Gelehrsamkeit mit der Buchführung der Kaufleute (Vine 2011), den Schreibstuben der Verwaltung (Siegert/Vogl 2003, Gardey 2008) oder den Begutachtungspraktiken der Rechtsprechung (Vismann 2011) teilte. Es ist gerade die Geschichte dieser kulturellen Praktiken, sozialen Räume und gesellschaftlichen Institutionen, auf die jene nicht beabsichtigten epistemischen Effekte des Aufschreibens und Ordnens aufmerksam machen und damit in Zusammenhang mit der Herausbildung und Formierung von Wissen bringen. Die hierbei in den Blick geratenden Schreibformen und -praktiken müssen, das ist ein weiterer Vorteil, nicht notwendig als „epistemische Genres“ (Pomata 2011) begriffen werden, sondern können dank ihrer formalen wie materialen Gestalt als allgemeine Genres in unterschiedliche soziale Welten zurückverfolgt werden.

Drei Felder werden in der neueren Forschung immer wieder genannt, die für die Erhebung, Speicherung und Verarbeitung von Informationen in der Frühen Neuzeit wichtig waren: humanistische Gelehrsamkeit (Waquet 2000), Verwaltung (Holenstein 2003) sowie Handel und Buchhaltung (Schäfer 2003). Die Bedeutung der hierbei verwendeten Praktiken für eine Wissensgeschichte, die über das reine information management (Behrisch 2008) hinausgehend den Wandel des Wissens selbst in den Blick nimmt, ist jedoch noch nicht geklärt. Hierzu müssen die fraglichen Praktiken genauer auf ihren Bezug zur Wissensproduktion betrachtet werden. Wenn das vorliegende Themenheft eine dieser Praktiken in den Mittelpunkt stellt, dann soll die Form des Sammelns und Ordnens von Notizen unter Schlagworten in erster Linie als Aufschreibepraktik begriffen werden, die auch - aber nicht ausschließlich der humanistischen Methode der Gemeinplätze (Loci communes oder common places) zu Grunde lag. Zur Anwendung kam diese Technik des Exzerpierens, Kopierens und Arrangierens nämlich nicht nur in den Stätten humanistischer Gelehrsamkeit wie Schulen oder Akademien. Man findet sie auch im Handel und in den frühen Formen staatlicher und städtischer Verwaltung sowie in der Rechtsprechung, ganz $\mathrm{zu}$ schweigen von ärztlichen Praxistagebüchern, wie Stolberg 2013 zeigt.

Die ubiquitäre Verbreitung verweist zugleich auf die Gefahr, sich spannenden Fragen zu verschließen, wenn man die Loci communes vorschnell auf eine der humanistischen Gelehrsamkeit eigene Methode reduziert. Vielmehr könnten die zeitgenössischen Bemühungen, die Natur nach Gemeinplätzen zu ordnen, selbst bereits als ein unbeabsichtigter Effekt dieser Papiertechnik aufgefasst und thematisiert werden, entspringen die Loci communes doch der rhetorischen Technik, vorab einen pragmatisch geordneten Vorrat an autoritativen Zitaten und Belegstellen für den Aufbau einer überzeugenden Argumentation bereitzustellen (Schmidt-Biggemann 1983). In methodischer Hinsicht wäre folglich nicht Plinius, sondern Cicero der legitime Vorfahre der Naturbeschreibungen des 17. und 18. Jahrhunderts. 
Was passiert, wenn eine Mnemotechnik zum Informationsmanagement herangezogen wird? Welche intendierten und unbeabsichtigten Folgen haben Exzerpieren, Loci communes und Papierschnipsel für das frühneuzeitliche Wissen der Natur und seine Ordnung entfaltet? Einige allgemeine Antworten geben die Studien von Zedelmaier 1992, Stammen/Weber 2004 und Blair 2010. Was aber waren die Folgen für den fachspezifischen Kanon des Naturwissens und für die Entwicklung einzelner Wissenschaften? Das ist eine dritte Perspektive, die eine Erforschung der paper technology eröffnet. In den bisherigen Analysen der humanistischen Schreib- und Mnemotechniken werden nämlich Fach- und Wissensgeschichte eher selten miteinander verknüpft, was weniger den Bemühungen beider Geschichtsschreibungen geschuldet ist, als einem offensichtlichen Gegensatz zwischen Buch und Natur.

\section{Fachgeschichte und Wissensgeschichte}

Die Geschichte der paper technology oder Papiertechniken ist, wie die gegenwärtige Forschung nahelegt, ein fruchtbarer Ansatz, um ein Dilemma der frühneuzeitlichen Wissenschaftsgeschichtsschreibung aufzulösen: den offenkundigen Gegensatz zwischen Bibliotheks- und Feldarbeit (Ogilvie 2006). Das Herausziehen und Festhalten bemerkenswerter Lesefrüchte ist ohne Frage eine ganz andere Aktivität als das Aufschreiben von Beobachtungen am Bett des Kranken, auf Almwiesen oder unter dem nächtlichen Firmament. Die gelehrten Humanisten trieben die Erforschung der Natur durch Textarbeit (die Erschließung, Bearbeitung und Zusammenstellung rezenter und antiker Schriften) in der Bibliothek ebenso voran wie durch eine immer umfangreichere Beobachtung der gegenständlichen Welt - sei es in der Wunderkammer (Daston/Park 1998), im Herbarium (Ogilvie 2006) oder auf dem Seziertisch (Cunningham 2010).

Aber wie verhalten sich beide Wissensräume zueinander? Papiertechniken helfen, die Kluft zwischen Buch und Natur, zwischen Bibliothek und Feld, $\mathrm{zu}$ überbrücken. Lesen und Beobachten weisen in ihrer humanistischen Ausprägung zwei durch Papiertechniken vermittelte Strukturmerkmale auf: die Dekontextualisierung sowie die Einübung moralischer Tugenden. Einerseits brachte das Aufschreiben sowohl die Früchte der Natur als auch die des Lesens in eine neue Anordnung, die es möglich machte, beide auf neue Weise zu sehen. Andererseits vermittelte die gemeinsame Aufschreibetechnik auch eine gemeinsame Haltung der Achtsamkeit und wurde damit Grundlage für eine „Kultur der Beobachtung“ (Daston 1988). In der Bibliothek wie in der Natur wurden die Mnemo- und Wissenstechniken eingeübt, eingeschliffen und weiterentwickelt. Im Hinblick auf die habituelle Haltung zielte hier das keineswegs flüchtige „Notiz nehmen“ auf mehr als nur das „Notizen machen“. 
$\mathrm{Zu}$ Recht hebt die neuere Forschung darauf ab, dass die humanistischen Textmethoden dazu beitrugen, eine empirische Weltsicht zu begründen oder gar einem empirischen Faktizismus den Weg zu bereiten (Blair 1992, 2005). Dieses Argument lässt sich aber noch präzisieren. Wie die Beiträge dieses Themenheftes nahelegen, lässt sich für diese Zeit kaum zwischen empirischer und humanistischer Methode trennen: Das Exzerpt einer tradierten Krankengeschichte galt ebenso als Beobachtung wie die eigenen Notizen am Krankenbett. In beiden Fällen lernte ein aufmerksamer, neugieriger, kritischer und potentiell unersättlicher Beobachter und Sammler etwas Neues über die Welt, erst recht, wenn er seine Aufschriebe wie Aldrovandi bearbeitete, nämlich anhäufte, kombinierte, gegenüberstellte, zusammenzog oder zerlegte. In dieser Hinsicht scheint die Arbeit am Schreibpult dem Experimentieren zu ähneln. In der Tat fühlt man sich, wie unser Beitrag über die Effekte des Auflistens bei François Boissier de Sauvages zeigt (Hess/Mendelsohn 2013), an eine Bemerkung von Ong (Blair 2005: 283) erinnert, der zufolge die Technik der common places nichts anderes als eine ,induction“ im baconischen Sinne sei. Um es deutlicher zu sagen: Der neue Empirismus der Fakten wurde nicht durch die humanistische Methode befördert im Sinne einer prädisponierenden Geisteshaltung. Es waren vielmehr die materialen Papiertechniken des Humanismus selbst, die zur empirischen Methode avancierten.

Mit dem Einbezug der paper technology stellt sich folglich erneut die Frage nach Status und Rolle der Methode für die Herausbildung eines Wissens von der Natur. Die nicht intendierten Effekte des physischen Hantierens und Arrangierens mit Aufschrieben und Textschnipseln waren möglicherweise ebenso wichtig wie die philosophische Methode, ob nun in aristotelischer, baconischer, cartesianischer oder gar ramistischer Tradition (Ong 2004, Hotson 2007). Es ist gerade das Verhältnis zwischen philosophischer Methode und materialen Techniken des Aufschreibens und Ordnens, aus dem für die Forschung spannende Fragen resultieren, ganz zu schweigen von den Wechselwirkungen und Querbezügen zu den sozialen Welten, in denen diese Papiertechniken ebenfalls zu Hause waren.

Diese Feststellung beantwortet jedoch nicht die Frage, welche Rolle die durch die Papiertechniken induzierten epistemischen Effekte bei der Entwicklung einzelner Wissenschaften gespielt haben. Die Feststellung, dass Ärzte oder Naturforscher, Studenten oder Gelehrte beim Aufschreiben und Ordnen ihrer Aufschriebe auf die gleichen Papiertechniken zurückgriffen und „Faktoide“ (Blair 1992) produzierten, trägt wenig dazu bei, zu verstehen, auf welche Weise hieraus so unterschiedliche disziplinäre Wissensformationen wie die Nosologie oder Systema naturae entstehen und erst recht sich eine Philosophia naturalis oder (als nicht mathematisierte Experimentalwissenschaft) die Chemie respektive Alchemie (Hannaway 1975) entwickeln konnten.

Die Autoren der Beiträge in diesem Heft versuchen - zumindest für einige Wissenschaftsbereiche - erste, vorsichtige Antworten zu geben. Dabei 
argumentieren sie, wie die Beispiele der ärztlichen Loci communes (Stolberg 2013) oder der nosologischen Klassifikation (Hess/Mendelsohn 2013) zeigen, nicht von einer Wissensgeschichte der frühneuzeitlichen oder humanistischen Papiertechniken ausgehend, sondern sie befragen gewissermaßen vom anderen Ende her die fachhistorischen narratives auf ihre Kontinuitäten und Brüche im Umgang mit Papier und Stift. Sie schauen kleinen und großen Helden wie Aldrovandi, Boissier de Sauvages und dem Linné-Schüler Willdenow (Böhme/Müller-Wille 2013) in dieser Geschichte erneut auf die Finger. Rasch zeigt sich hierbei, dass der Vorgang des Aufschreibens und Ordnens keineswegs auf eine bloße Speicherung und Verwaltung von Informationen reduziert werden kann. Auch werden mit diesen Praktiken nicht „rohe Informationen“ in einen verdaulichen Zustand gebracht, wie Burke (2000: 11) diesen Vorgang in seiner populären Kulturgeschichte des Wissens dargestellt hat. Vielmehr sind es solche Papiertechniken selbst, die Informationen in fachspezifisches Wissen verwandeln: erstens durch die Autorisierung eigener Beobachtungen, die unter den Loci communes ärztlicher Tagebücher unterschiedslos neben der Meinung überlieferter Autoritäten (Stolberg 2013) stehen. Zweitens führen Aufschreiben und Ordnen aber auch zur Generalisierung partikulärer Beobachtungen - ob nun in der Abgrenzung von Krankheiten als „Wesenheiten“ (ebd.) oder der Einordnung der Brandenburger Flora (Böhme/Müller-Wille 2013). Drittens erzeugen diese Papiertechniken eine Ordnung der fachwissenschaftlichen Dinge, seien es nun die mühsam mit Leim und Schere zu bändigenden Folianten des italienischen Naturforschers Aldrovandi (Krämer 2013) oder die in immer neuen Revisionen publizierten Klassifikationssysteme von Linné (Müller-Wille/Scharf 2011) und Sauvages (Hess/Mendelsohn 2013). Die Beiträge dieses Themenheftes tragen insofern dazu bei, das weite Feld der epistemischen Effekte und Wirkungen solcher Papiertechniken zu sondieren. Die Wissensgeschichte der paper technology ist jedoch noch zu schreiben.

\section{Anmerkungen}

1 Zur Technik der Verzettelung siehe Zedelmaier 1992, Meinel 1995 und Krajewski 2002.

2 Einen Überblick bietet das von Blair und Yeo 2010 herausgegebene Themenheft der Zeitschrift Intellectual History Review sowie der 2011 erschienene Sammelband von Daston und Lunbeck. Bemerkenswert ist auch die systematische Exploration des Feldes durch den Münchener Sonderforschungsbereich „Pluralisierung und Autorität“, insbesondere die Sammelbände von Büttner/Friedrich/Zedelmaier 2003, Brendecke/Friedrich/ Friedrich 2008, Schierbaum 2009 und Müller/Oesterreicher/Vollhardt 2010. Für die Naturgeschichte siehe Grafton/Siraisi 1999 und Park 2011. In diesem Zusammenhang sollte man auch nicht die Beschäftigung mit Notiz- und Laborbüchern vergessen, auch wenn der Schwerpunkt hier meistens in der Moderne liegt: Holmes/Renn/Rheinberger 2003, Hoffmann 2008.

3 Vgl. hierzu Hess/Mendelsohn 2010. Die Rolle der paper technology in der Genese 
ärztlichen Wissens steht momentan im Mittelpunkt eines größeren Forschungsvorhabens, das vom Europäischen Forschungsrat gefördert wird (http://www.papertechnology.org, letzter Zugriff am 5. Dezember 2012). Für ihre hilfreiche Kritik danken wir Alexa Geisthövel, Staffan Müller-Wille, Ruth Schilling und Michael Stolberg sowie den unbekannten Gutachterinnen und Gutachtern der NTM.

4 Der Begriff wurde ursprünglich im Englischen geprägt, weshalb er sich kaum übersetzen lässt: Der Begriff technology ist viel konkreter als das deutsche Abstraktum „Technologie“, weshalb wir im Deutschen den Begriff Papiertechniken im Plural bevorzugen und die einzelnen Verfahren mit dem Singular bezeichnen.

5 Siehe hierzu insbesondere Eisenstein 1979 und die daran anknüpfende Debatte bei Johns 1998 und Baron/Lindquist/Shevlin 2007.

\section{Literatur}

Baron, Sabrina Alcorn/Lindquist, Eric N./Shevlin, Eleanor, Hg., 2007. Agent of Change. Print Culture Studies after Elizabeth L. Eisenstein. Amherst [u. a.]: University of Massachusetts Press.

Behrisch, Lars, 2008. Zu viele Informationen! Die Aggregierung des Wissens in der Frühen Neuzeit. In: Arndt Brendecke, Markus Friedrich und Susanne Friedrich, Hg., Information in der Frühen Neuzeit. Status, Bestände, Strategien. Berlin: LIT Verlag, 455-473.

Blair, Ann, 1992. Humanist Methods in Natural Philosophy. The Commonplace Book. Journal of the History of Ideas, 53, 541-551.

Blair, Ann, 2005. Historia in Zwinger's Theatrum humanae vitae. In: Gianna Pomata und Nancy G. Siraisi, Hg., Historia. Empiricism and Erudition in Early Modern Europe. Cambridge, Mass./ London: MIT Press, 269-296.

Blair, Ann, 2010. Too Much to Know. Managing Scholarly Information before the Modern Age. New Haven: Yale University Press.

Blair, Ann/Yeo, Richard, Hg., 2010. Note-Taking in Early Modern Europe. Intellectual History Revue, 20, 301-433.

Böhme, Katrin/Müller-Wille, Staffan 2013. „In der Jungfernheide hinterm Pulvermagazin frequens". Das Handexemplar des Florae Berolinensis Prodromus (1787) von Karl Ludwig Willdenow. NTM. Zeitschrift für Geschichte der Wissenschaften, Technik und Medizin. N. S., 21, im gleichen Heft.

Brendecke, Arndt/Friedrich, Markus/Friedrich, Susanne, Hg., 2008. Information in der Frühen Neuzeit. Status, Bestände, Strategien. Berlin: LIT Verlag.

Burke, Peter, 2000. A Social History of Knowledge. From Gutenberg to Diderot. Cambridge: Polity Press, Blackwell Publishers.

Büttner, Frank/Friedrich, Markus/Zedelmaier, Helmut, 2003. Sammeln, Ordnen, Veranschaulichen. Zur Wissenskompilatorik in der Frühen Neuzeit. Münster: LIT.

Cunningham, Andrew, 2010. The Anatomist Anatomis'd. An Experimental Discipline in Enlightenment Europe. Farnham: Ashgate.

Daston, Lorraine, 1988. The Factual Sensibility (Review). Isis, 79, 452-467.

Daston, Lorraine/Lunbeck, Elizabeth, Hg., 2011. Histories of Scientific Observation. Chicago/ London: The University of Chicago Press.

Daston, Lorraine/Park, Katharine, 1998. Wonders and the Order of Nature, 1150-1750. New York: Zone Books.

Dear, Peter, Hg., 1991. The Literature Structure of Scientific Argument. Historical Studies. Philadelphia: University of Pennsylvania Press.

Dear, Peter, 1985. Totius in Verba. Rhetoric and Authority in the Early Royal Society. Isis, 76, $145-161$.

Edgerton, Samuel Y., 1980. The Renaissance Artist as Quantifier. In: Margaret A. Hagen, Hg., The Perception of Pictures I. Alberti's Window. The Projective Model of Pictures. New York: Academic Press, 179-212.

Eisenstein, Elisabeth, 1979. The Printing Press as an Agent of Change. Communication and Cultural Transformations in Early-Modern Europe. Cambridge: Cambridge University Press. 
Gardey, Delphine, 2008. Ecrire, Calculer, Classer. Comment une Révolution de Papier a Transformé les Sociétés Contemporaines (1800-1940). Paris: Découverte.

Grafton, Anthony T./Siraisi, Nancy G., Hg., 1999. Natural Particulars. Nature and the Disciplines in Renaissance Europe. Cambridge, Mass./London: MIT Press.

Hannaway, Owen, 1975. The Chemists and the Word. The Didactic Origins of Chemistry. Baltimore: Johns Hopkins University Press.

te Heesen, Anke, 2005a. Accounting for the Natural World. Double-Entry Bookkeeping in the Field. In: Londa Schiebinger und Claudia Swan, Hg., Colonial Botany. Science, Commerce, and Politics in the Early Modern World. Philadelphia: University of Pennsylvania Press, 237-251.

te Heesen, Anke, 2005b. The Notebook. A Paper-Technology. In: Bruno Latour und Peter Weibel, Hg., Making Things Public. Atmospheres of Democracy. Cambridge, Mass./London: MIT Press, 582-589.

Hess, Volker/Mendelsohn, J. Andrew, 2010. Case and Series. Medical Knowledge and Paper Technology, 1600-1900. History of Science, 48, 287-314.

Hess, Volker/Mendelsohn, J. Andrew 2013. Fallgeschichte, Historia, Klassifikation. François Boissier de Sauvages bei der Schreibarbeit. NTM. Zeitschrift für Geschichte der Wissenschaften, Technik und Medizin. N. S., 21, im gleichen Heft.

Hoffmann, Christoph, Hg., 2008. Daten sichern. Schreiben und Zeichnen als Verfahren der Aufzeichnung. Zürich, Berlin: diaphanes.

Holenstein, André, 2003. „Gute Policey“ und lokale Gesellschaft im Staat des Ancien Régime. Das Fallbeispiel der Markgrafschaft Baden (-Durlach). Epfendorf: Bibliotheca Academica-Verlag.

Holmes, Frederic Lawrence/Renn, Jürgen/Rheinberger, Hans-Jörg, Hg., 2003. Reworking the Bench. Research Notebooks in the History of Science. Dordrecht [u. a.]: Kluwer Academic Publishers.

Hotson, H., 2007. Commonplace Learning. Ramism and its German Ramifications, 1543-1630. New York: Oxford University Press.

Johns, Adrian, 1998. The Nature of the Book. Print and Knowledge in the Making. Chicago/London: University of Chicago Press.

Klein, Ursula, 2001. Paper Tools in Experimental Cultures. Studies in History and Philosophy of Science, 32, 265-302.

Krajewski, Markus, 2002. Zettelwirtschaft. Die Geburt der Kartei aus dem Geiste der Bibliothek. Berlin: Kulturverlag Kadmos.

Krämer, Fabian 2013. Ein papiernes Archiv für alles jemals Geschriebene. Ulisse Aldrovandis Pandechion Epistemonicon und die Naturgeschichte der Renaissance. NTM. Zeitschrift für Geschichte der Wissenschaften, Technik und Medizin. N. S., 21, im gleichen Heft.

Krämer, Sybille, 1988. Symbolische Maschinen. Die Idee der Formalisierung in geschichtlichem Abriß. Darmstadt: Wissenschaftliche Buchgesellschaft.

Kusukawa, Sachiko, 2012. Picturing the Book of Nature. Image, Text, and Argument in SixteenthCentury Human Anatomy and Medical Botany. Chicago/London: Chicago University Press.

Lynch, Michael/Woolgar, Steve, Hg., 1990. Representation in Scientific Practice. Cambridge, Mass./London: MIT Press.

Mahoney, Michael S., 1985. Diagrams and Dynamics. Mathematical Perspectives on Edgerton's Thesis. In: John William Shirley und F. David Hoeniger, Hg., Science and the Arts in the Renaissance. Washington, D.C.: Folger Shakespeare Library, 198-220.

Meinel, Christoph, 1995. Enzyklopädie der Welt und Verzettelung des Wissens. Aporien der Empirie bei Joachim Jungius. In: Franz M. Eybl, Wolfgang Harms und Hans-Henrik Krummacher, Hg., Enzyklopädien der Frühen Neuzeit. Beiträge zu ihrer Forschung. Tübingen: Max Niemeyer, 162-187.

Müller, Jan-Dirk/Oesterreicher, Wulf/Vollhardt, Friedrich, Hg., 2010. Pluralisierungen. Konzepte zur Erfassung der Frühen Neuzeit. Berlin: De Gruyter.

Müller-Wille, Staffan/Scharf, Sara, 2011. Indexing Nature. Carl Linnaeus and his Fact Gathering Strategies. Svenska Linnesällskapets Årsskrift, 31-60.

Ogilvie, Brian W., 2006. The Art of Describing. Natural History in Renaissance Europe. Chicago/ London: The University of Chicago Press.

Ong, Walter J., 2004. Ramus, Method, and the Decay of Dialogue: From the Art of Discourse to the Art of Reason (1958). Chicago/London: The University of Chicago Press.

Park, Katharine, 2011. Observation in the Margins, 500-1500. In: Lorraine Daston und Elizabeth Lunbeck, Hg., Histories of Scientific Observation. Chicago/London: The University of Chicago Press, $15-44$. 
Pomata, Gianna, 2011. Observation Rising. Birth of an Epistemic Genre, 1500-1650. In: Lorraine Daston und Elizabeth Lunbeck, Hg., Histories of Scientific Observation. Chicago/ London: The University of Chicago Press, 45-80.

Rusnock, Andrea A., 2002. Vital Accounts. Quantifying Health and Population in EighteenthCentury England and France. Cambridge: Cambridge University Press.

Schäfer, Ernesto, 2003. El Consejo real y Supremo de las Indias. Su Historia, Organización y Labor Administrativa hasta la Terminación de la Casa de Austria: Historia y Organización del Consejo y de la Casa de la Contratación de las Indias. Madrid.

Schierbaum, Martin, Hg., 2009. Enzyklopädistik 1550-1650. Typen und Transformationen von Wissensspeichern und Medialisierung des Wissens. Berlin: LIT Verlag.

Schmidt-Biggemann, Wilhelm, 1983. Topica universalis. Eine Modellgeschichte humanistischer und barocker Wissenschaft. Hamburg: Felix Meiner.

Shapin, Simon, 1984. Pump and Circumstance. Robert Boyle's Literary Technology. Social Studies of Science, 14, 481-521.

Siegert, Bernhard/Vogl, Joseph, Hg., 2003. Europa. Kultur der Sekretäre. Zürich/Berlin: diaphanes.

Soll, Jacob, 2010. From Note-Taking to Data Banks. Personal and Institutional Information Management in Early Modern Europe, Intellectual History Review, 20, 355-375.

Stammen, Theo/Weber, Wolfgang E.J., Hg., 2004. Wissenssicherung, Wissensordnung und Wissensverarbeitung. Das Europäische Modell der Enzyklopädien. Berlin: Akademie Verlag.

Stolberg, Michael 2013. Medizinische Loci communes. Formen und Funktionen einer ärztlichen Aufzeichnungspraxis im 16. und 17. Jahrhundert. NTM. Zeitschrift für Geschichte der Wissenschaften, Technik und Medizin. N. S., 21, im gleichen Heft.

Vine, Angus, 2011. Commercial Commonplacing. Francis Bacon, the Waste-Book, and the Ledger. In: Richard Beadle und Colin Burrow, Hg., Manuscript Miscellanies c. 1450-1700. London: The British Library, 197-218.

Vismann, Cornelia, 2011. Medien der Rechtsprechung. Frankfurt a. M.: S. Fischer.

Waquet, Françoise, Hg., 2000. Mapping the World of Learning. The Polyhistor of Daniel Georg Morhof. Wiesbaden: Harrassowitz.

Warwick, Andrew C., 2003. Masters of Theory. Cambridge and the Rise of Mathematical Physics. Chicago/London: The University of Chicago Press.

Wübben, Yvonne, 2009. Ordnen und Erzählen. Emil Kraepelins Beispielgeschichten. Zeitschrift für Germanistik, 14, 381-395.

Zedelmaier, Helmut, 1992. Bibliotheca Universalis und Bibliotheca Selecta. Das Problem der Ordnung des gelehrten Wissens in der Frühen Neuzeit. Köln u. a.: Böhlau.

\section{Volker Hess}

Institut für Geschichte der Medizin

Charité Universitätsmedizin Berlin

Ziegelstraße 10

10117 Berlin

Deutschland

E-mail: volker.hess@charite.de

\section{J. Andrew Mendelsohn}

School of History

Queen Mary, University of London

London

E1 4NS

\section{United Kingdom}

E-mail: a.mendelsohn@qmul.ac.uk 